\title{
Living together, feeding apart: How to measure individual food consumption in social house mice
}

\author{
FRIEDER NEUHÄUSSER-WESPY and BARBARA KÖNIG \\ University of Zurich, Zurich, Switzerland
}

\begin{abstract}
In many studies with animals kept in groups, scientists need information about each individual's food access without disturbance or separation of the animals. We developed an automatic feeding device that allows measurement of individual food consumption and experimental manipulation of individual food availability in small social mammals, such as house mice. The feeding device is based on radio frequency identification that triggers access to a motor-driven metal arm filled with food pellets and is mediated with the help of subcutaneously implanted transponders.
\end{abstract}

The ability to measure and manipulate individual food consumption is of paramount importance for many topics involving animal research in ethology, psychology, and physiology. Although this is a rather easy task when animals are kept solitarily, it often raises problems with social species kept in groups. Here, individual food consumption typically can only be measured with some interference by the experimenter and, thus, disturbance of the animals. To avoid such problems, we developed an automatic feeding device that allows unobtrusive, separate, and exclusive food access for several individuals living in a social group.

We have used this device to study the maternal investment of female house mice that cooperatively rear their young in a communal nest and indiscriminately nurse their own and alien young (see, e.g., König, 1989a, 1989b, 1994b).

\section{Maternal Investment in \\ Communally Nursing House Mice}

As in most mammals, milk production is a large investment for a mouse mother (Bronson, 1989; CluttonBrock, 1991). Milk production is costly not only in terms of energy, but also in terms of decreased future reproduction (König, Riester, \& Markl, 1988). With increased litter size and correspondingly increased milk production, the birth of the female's next litter is delayed. Because of such costs, we expect females that share a communal nest to mutualistically nurse nonoffspring. As a

The project is financially supported by the Swiss National Science Foundation (Nr. 31-507 40.97). We sincerely thank Norbert Schneider (Würzburg) for constructing the mechanical parts of the device. For kind support and cooperation, we very much thank J. Rohrmüller and P. Kribitzneck at Micromedia AG. We very much thank D. C. Turner for correcting the English and two anonymous reviewers for helpful comments. Correspondence concerning this article should be addressed to F. Neuhäusser-Wespy, University of Zurich, Institute of Zoology, Winterthurerstr. 190, CH-8057 Zurich, Switzerland (e-mail: fneuhaeu@ zool.unizh.ch). consequence, milk production supposedly plays a crucial role in stabilizing cooperation among communally nesting female house mice (König, 1994a, 1997).

To analyze such altruistic cooperation (as defined by Hamilton, 1964), we intend first to quantify the energy spent for milk production during lactation. This can be done by measuring the daily food consumption by a mother from the day of birth of a litter until weaning. Second, we intend to experimentally manipulate the amount of milk available to the pups in order to study the behavior of two cooperating females under limiting conditions. This can be done by restricting one or both of the females' access to food, which causes reduced milk production (König, 1989b).

In both cases, we face the problem that, in a social group, all the group members usually have access to all parts of their common environment and to any food available. However, we need a laboratory setting in which everything except the food can be shared by all of the group members. Thus, we have developed a technical solution that allows separate control of access to food for each individual.

\section{Technical Approach to Solving the Problem}

The solution to our problem can be divided into two steps. First, group members must be discriminated, which means that they have to be individually marked to allow identification. Such identification has to be automatic, without any disturbance of the animals. In the second step, successful identification must generate an output that will give access to food. Naturally, the solution can be applied for other purposes as well.

There are several technical solutions for automatically identifying animals, but only a few are suitable for small rodents and our specific requirements, which are as follows.

First, the technique has to provide a true identification and not just a binary decision, such as has a marker or not. Binary detection would imply that, after an unsuccessful reading, an individual would simply be treated as an unmarked group member. Our feeding device, however, should only react after successful identification. 
Second, the markers have to be small and light (less than $1 \mathrm{~g}$ ), owing to the small size of the animals. The markers should be implantable, because everything attached to the animal (ears, legs, or skin) or glued onto the fur can be chewed or torn off. Furthermore, an injectable marker would avoid surgical procedures.

Third, identification and discrimination should be possible over a distance of several centimeters and must have a low error rate.

Finally, the technique has to be inexpensive, since one experimental setup will require several of these devices (depending on group size).

A well-established technique that fulfills all of these requirements is electronic identification with transponders, also called radio frequency identification (RFID). Transponders of this type are used for a variety of applications-among others, for marking animals (see, e.g., Kerth \& König, 1996). A number of suppliers produce implantable transponders for marking experimental animals or domestic pets; these are often sold in sterile needles that allow easy injection under the skin of an animal (Figure 1). Today, glass-encapsuled transponders allow speedy tracing of the origin or the owner of lost animals by reference to a central database. In farm animals-for example, cattle-implanted transponders are replacing branding and tattooing, and transponder-based feeding systems that can feed animals individually are already in use.

Such transponders work without a battery. In principle, they are magnetic coupled devices with antenna systems that are designed to collect energy from the magnetic field of a specific reading device. The energy loads a capacitor, which supplies the transponder chip with electric energy. The activated chip then transmits its unique code by switching an additional capacitor, bit by bit, on and off; this, in turn, alters the properties of the resonant circuit and, thus, the responding frequency. The result is a frequency-shifted signal that contains the individual code of the transponder (Figure 2). The signal is received by the antenna of the reading device, where it is then demodulated and decoded, and the code of the transponder is displayed on the reading device or sent to a computer.

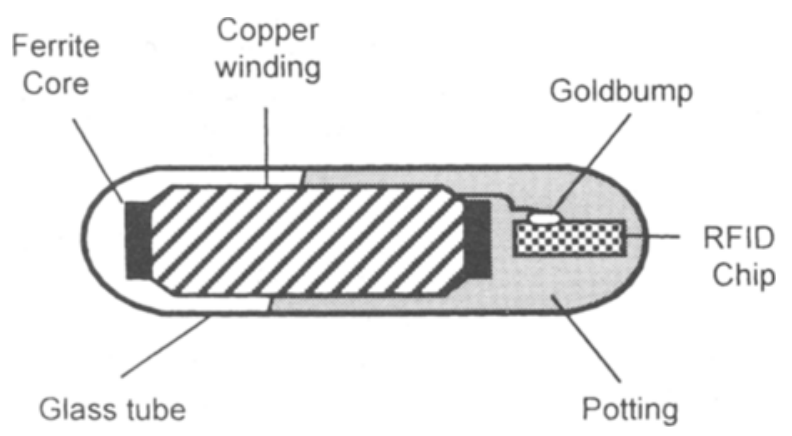

Figure 1. Schematic drawing of a glass-encapsuled transponder (weight, $0.1 \mathrm{~g}$; length, $12 \mathrm{~mm}$; diameter, $2.1 \mathrm{~mm}$ ) that can be subcutaneously implanted for permanent individual identification of experimental animals.
Most of the reading devices used for this purpose are handheld readers or stationary readers with custommade antenna and are fairly expensive (more than $\$ 500$ per device). However, Micromedia AG (Hallbergmoos, Germany) produces a small all-in-one reader, Easy Key, that was originally designed for applications such as electronic time registration, access control, or car immobilizers. All components and electronics are located on a small circular board (diameter, $80 \mathrm{~mm}$; height, $25 \mathrm{~mm}$ ), with an integrated antenna, allowing low-priced production (the price for a single unit is less than $\$ 100$ ). The readers have to be supplied with $5 \mathrm{~V} \mathrm{DC}$ (power consumption, $35 \mathrm{~mA}$ ) and can also be driven with an external antenna.

It is possible to operate the Easy Key reader together with small glass-encapsuled transponders $(12 \times 2.1 \mathrm{~mm}$, $0.1 \mathrm{~g}$ ) based on the H4001-Chip (EM MicroelectronicMarin SA). These transponders, however, are incompatible with the standards that are often in use in animal research (such as Trovan, Destron, Avid, ISO 1 1784). They come from the factory with a unique 10-digit code number that cannot be altered by the user. The transponders can be injected under the skin of a mouse with a medical syringe. Subcutaneous wandering of transponders is rare and does not interfere with identification (personal observations).

Reading distance principally depends on the size of a transponder; with our transponders, successful reading was obtained only within $10 \mathrm{~mm}$. Inside of the Easy Key antenna (diameter, $50 \mathrm{~mm}$ ), however, reading is always successful, even in the disadvantageous situation in which the orientation of the transponder is $45^{\circ}$ to the lines of force of the antenna's field.

Two types of readers are offered by Micromedia AG that are of interest for behavioral studies. The Easy Key/r has an RS232 interface that allows direct communication with the serial port of a PC. The producer also supplies the software necessary to document the code and time of transponder identifications.

The Easy Key/s has no RS232 interface but has a small memory chip that can be programmed with the codes of 1 to 117 transponders. A relay that is integrated on the board will only switch if one of the previously stored codes is identified by the reader. The reading of a transponder whose code is not stored will cause no action. Programming is simple and done directly on the board itself. The codes remain in the memory even if the power is disconnected. We have chosen the Easy Key/s because it provides us with a stand-alone solution that can work without an additional computer. With the help of two (or more) of these readers, with one transponder code stored on each, it is possible to control two (or more) automatic feeding devices that allow access to only one individual each.

\section{The Mechanical Feeding Device}

Our mechanical feeding devices were developed at the University of Würzburg, Germany. Each consists of a movable hollow metal arm filled with commercial food 


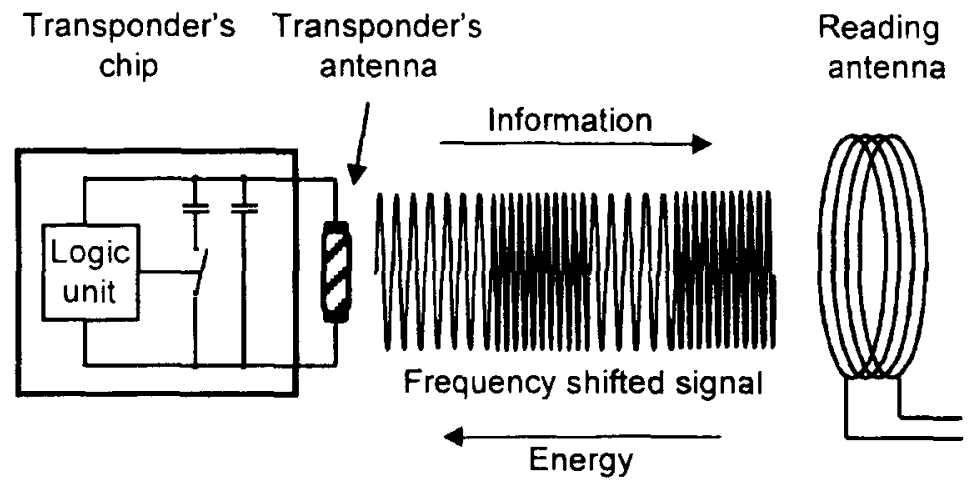

Figure 2. Data transfer from transponder (left) to reader (right) with frequency-shifted modulation. See the text for further explanation.

pellets (Figure 3 ). In the case of correct identification, the relay of the reader will switch and give power to a small servomotor that moves the metal arm into close contact with the lid of the cage, so that the animal can feed on the pellets as long as it remains in the feeding station within the field of the circular antenna. Daily food consumption is measured by weighing and, if necessary, refilling the pellets in each metal arm every $24 \mathrm{~h}$.

In experiments under restricted feeding, it is important to prevent the animals from stealing food from other group members. A small wire at the end of the metal arm prevents the pellets from sliding out, and the mice can only grab relatively small pieces. As a consequence, most feeding takes place inside of the feeding station. If a second animal closely approaches a feeding individual, the second transponder induces the metal arm to move away from the cage lid, since the reader cannot identify the transponder (and thus allow successful reading) if two animals are simultaneously in the field of the antenna. Therefore, a second animal may disturb the first one dur-

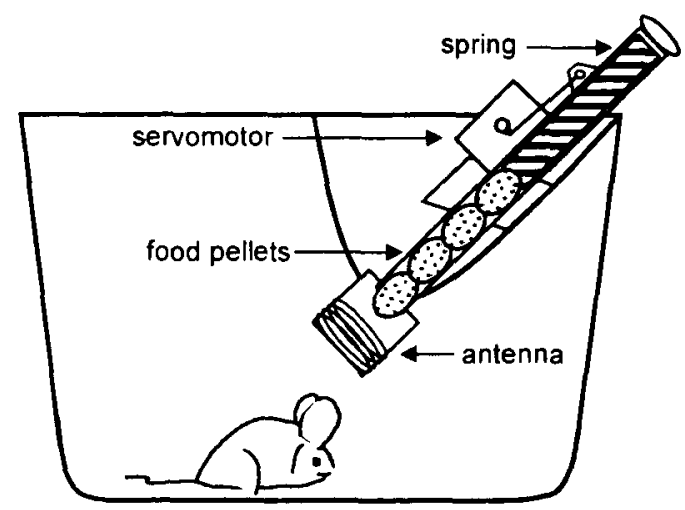

Figure 3. Schematic drawing of a macrolon cage with our automatic feeding device. If a house mouse with the correct transponder code raises its body into the field of the circular antenna, the servomotor will move the metal arm filled with food pellets to the lid of the cage so that the animal can feed as long as it remains inside the feeding device. ing feeding, but stealing a significant amount of food is impossible.

\section{Potential for Further Research}

Unobtrusive, automatic identification of animals with RFID is very useful for behavioral research. The technique has been available for many years but has rarely been used in experimental studies for which many reading devices were needed, because of its high price. The Easy Key/s is a convenient stand-alone solution that can allow specific access to resources such as food, water, nesting sites, or mating partners.

The device presented here can also be used to register the presence of individual small rodents, birds, or bats in their nest boxes, at feeding sites, or in other preferred places. We have further used the device to study the behavior of small rodents living in seminatural enclosures. The tubelike entrances of all nest boxes are equipped with an antenna (Easy Key) that records (in real time) the code of every individual entering or leaving a box. This allows automatic documentation of the distribution of all the members of a population in time and space, which is a prerequisite for understanding the species' social behavior. Such an identification technique can also be combined with a balance (placed, e.g., under the entrance to a nest box or under a water bottle) to automatically register individual body weight.

The combination of automatic identification with biotelemetric data, such as body temperature or heart rate measured with the help of small implantable chips (which may be possible in the near future), will allow for improved access to physiological data in behavioral research, even with animals kept in groups.

\section{REFERENCES}

Bronson, F. H. (1989). Mammalian reproductive biology. Chicago: University of Chicago Press.

Clutton-Brock, T. H. (1991). The evolution of parental care, Princeton, NJ: Princeton University Press.

Hamilton, W. D. (1964). The genetical evolution of social behaviour (I and II). Journal of Theoretical Biology, 7, 1-52. 
KERTH, G., \& KöNIG, B. (1996). Transponder and infrared-videocamera as methods used in a field study on the social behaviour of Bechstein's bats (Myotis bechsteini). Myotis, 34, 27-34.

KöNIG, B. (1989a). Behavioural ecology of kin recognition in house mice. Ethology, Ecology, \& Evolution, 1, 99-110.

KöNIG. B. (1989b). Kin recognition and maternal care under restricted feeding in house mice (Mus domesticus). Ethology, 82, 328-343.

KöNIG, B. (1994a). Communal nursing in mammals. Verhandlungen der Deutschen zoologischen Gesellschaft, 87, 115-127.

KöNIG, B. (1994b). Components of lifetime reproductive success in com- munally and solitarily nursing house mice: A laboratory study. Behavioural Ecology \& Sociobiology, 34, 275-283.

KöNIG, B. (1997). Cooperative care of young in mammals. Naturwissenschaften, 84, 95-104.

KÖNIG, B., Riester, J., \& MARKL, H. (1988). Maternal care in house mice (Mus musculus): II. The energy cost of lactation as a function of litter size. Journal of Zoology, 216, 195-210.

(Manuscript received March 10, 1999;

revision accepted for publication October 3, 1999.) 